\title{
COMPORTAMIENTO INGESTIVO DE VACAS LECHERAS PASTOREANDO LOLIUM PERENNE L. CON DIFERENTE TIPO Y NIVEL DE NEOTYPHODIUM LOLII (LATCH, CHRISTENSEN Y SAMUELS).
}

\section{INGESTIVE BEHAVIOUR OFDAIRY COWSGRAZINGLOLIUM PERENNE L. PASTURES WITH DIFFERENT TYPES AND LEVELS OF NEOTYPHODIUM LOLII (LATCH, CHRISTENSEN AND SAMUELS).}

Carlos A. Soto S., Ignacio F. López C. y Oscar A. Balocchi L.

Instituto de Producción Animal, Facultad de Ciencias Agrarias, Universidad Austral de Chile. Casilla 567, Valdivia., ilopez@uach.cl.

\section{RESUMEN}

Palabras claves: Hongo endófito, pastoreo, comportamiento ingestivo, preferencia.

Se evaluó el comportamiento ingestivo de vacas lecheras al pastorear praderas de $L$. perenne cv. Quartet, que contenían diferentes tipos y niveles de hongo endófito. El ensayo se realizó en Osorno, en praderas de $L$. perenne cv. Quartet con los siguientes tratamientos: T1: L. perenne con bajo endófito (BES), T2: $L$. perenne AR1 y T3: $L$. perenne con alto endófito (AES). Las parcelas fueron de 160 $\mathrm{m}^{2}$ y se pastorearon con 20 vacas lecheras. Se midió la disponibilidad de materia seca y componentes del rendimiento de los macollos antes y después de cada pastoreo. También se midió número de macollos, preferencia en pastoreo, comportamiento ingestivo en pastoreo y consumo aparente. El estudio se ajustó a un diseño de bloques completos al azar con tres tratamientos y tres repeticiones. Se aplicaron los análisis de varianza (ANDEVA) y análisis de variables canónicas (AVC). No hubo diferencias en los atributos de la pradera antes del pastoreo. Después del pastoreo el largo de lámina fue menor para Quartet AES y AR1 lo que coincide con un mayor consumo aparente para estos tratamientos. El Análisis de Variables Canónicas indicó que las diferencias entre tratamientos fueron explicadas por la disponibilidad de

\section{ABSTRACT}

Key words: endophyte fungi, grazing, ingestive behaviour, preference.

The ingestive behaviour of dairy cows grazing L. perenne cv. Quartet with different levels and types of endophyte was evaluated over a 5 -month period (Spring-Autumn). The trial was carried out in Osorno, on pastures of $L$. perenne cv. Quartet with: Low levels of endophytes (BES); endophyte AR1; and high levels of endophytes (AES). The pastures occupied individual plots of $160 \mathrm{~m}^{2}$ and were grazed by 20 dairy cows. The evaluated variables in pre and post grazing were herbage mass and the components of the yield of individual tillers. During grazing, bite number per minute and grazing preference were assessed. After grazing, tiller density and apparent intake of herbage mass were measured. The study corresponded to a Complete Randomized Block design with three blocks. ANOVA and Canonical Variate Analysis (CVA) were applied. Growth features between cultivars were similar. The remaining lamina length after grazing was smaller for Quartet AES and AR1 in relation to Quartet BES, which was consistent with the higher apparent intake of herbage mass for these treatments. The CVA showed that the largest differences between treatments were explained by available herbage mass before and after 
materia seca antes y después del pastoreo y largo de lámina antes del pastoreo. Las vacas lecheras discriminaron en el pastoreo entre los tratamientos mostrando una mayor preferencia y consumo de Quartet AR1 y AES.

\section{INTRODUCCIÓN}

La presencia de Neotyphodium lolii, el hongo endófito de las ballicas (Glenn y Bacon, 1997) en Lolium perenne $\mathrm{L}$. previene ataques de plagas de insectos como Listronotus bonariensis (gorgojo argentino del tallo). Neotyphodium lolii produce alcaloides con características toxicas como lolitrem B y ergovalina, los que pueden causar problemas de salud en el ganado, a través de los cuadros de "temblor muscular" o el "estrés por calor", respectivamente (Fletcher y Harvey, 1981; Easton et al., 2001; Rattray, 2003).

Neotyphodium lolii también produce el alcaloide peramina, el que posee acción insecticida y es inocuo para el ganado (Steward, 1985; Ball et al., 1993). Estudios realizados en Nueva Zelandia compararon el comportamiento de $L$. perenne con y $\sin N$. lolii mostraron que $L$. perenne sin endófito fue severamente dañada por $L$. bonaeriensis (Prestidge et al., 1982). Casos similares han sido reportados por Mortimer y Di Menna (1983), Prestidge y Ball (1993) y Popay y Rowan (1994), en los que se muestra que $N$. lolii es importante en asegurar la persistencia de L. perenne.

El aislamiento e inoculación de cepas de $N$. lolii en $L$. perenne obtuvo nuevos tipos del hongo, capaces de generar altos niveles de peramina, pero con una nula presencia de lolitrem B y ergovalina, llamado endófito AR1 (Wrightson Seeds, 2007).

La secreción de alcaloides al medio intercelular le confiere a las plantas de $L$. perenne recibir un menor daño por el gorgojo y una mayor tolerancia al estrés hídrico de verano (Easton $e t$ al., 2001; Rattray, 2003), es decir, permite a las plantas de $L$. perenne que lo poseen mantenerse más sanas y activas durante el año. Sin embargo, se ha observado desde el punto de vista de los animales, la presencia del hongo endófito, unida grazing and the lamina length before grazing. Grazing cows discriminated between the type of $L$. perenne according to its endophyte and season: cv. Quartet BES was preferred during November and December, cv. Quartet AR1 was preferred in January and during March the cows did not discriminate between the different types of cv. Quartet.

a los alcaloides que produce, puede generar rechazo en el consumo de estas plantas de $L$. perenne (Fletcher, 1999). Se han realizado estudios contrastando diferentes cultivares de L. perenne entre sí (Bluett et al., 2005), pero no se ha evaluado el efecto de los diferentes tipos de hongo endófito sobre el comportamiento ingestivo de los animales a pastoreo.

El objetivo del presente estudio fue evaluar el comportamiento ingestivo de vacas lecheras al pastorear un mismo cultivar de $L$. perenne pero con diferentes niveles de hongo endófito (endófito estándar en nivel bajo o alto), o con un tipo diferente de hongo endófito, como es el caso de AR1.

\section{MATERIALES Y MÉTODO}

El estudio se realizó desde la primavera de 2005 (Noviembre) a inicios de otoño del 2006 (Marzo), en Osorno, zona con una pluviometría anual de 1200 a $1500 \mathrm{~mm}$, con $400-500 \mathrm{~mm}$ en otoño, $700 \mathrm{~mm}$ en invierno, $250-400 \mathrm{~mm}$ en primavera y $150-200 \mathrm{~mm}$ en verano (Chile, INIA, 1985).

El suelo es trumao del Llano Central, serie Osorno, con topografía plana a ondulada, plano fluvio glacial, de desarrollo incipiente, con buen drenaje y textura media (Chile, INIA, 1985).

Durante la primavera de 2004 se sembraron parcelas de $20 \times 8 \mathrm{~m}$ de Lolium perenne cv. Quartet que diferían en el tipo o nivel de endófito presente. Los tratamientos fueron: T1: L. perenne cv. Quartet con bajo nivel de endófito estándar (BES); T2: L. perenne cv. Quartet con endófito AR1; y T3: L. perenne cv. Quartet con alto nivel de endófito estándar (AES).

Para el control de malezas de hoja ancha se aplicó MCPA en dosis de 560 g i.a. ha ${ }^{-1}$ en mezcla con clopiralid en dosis de $95 \mathrm{~g}$ i.a. ha 
${ }^{1}$, disueltos en $150 \mathrm{~L}$ agua antes del inicio del estudio .

La fertilización anual fue de $84 \mathrm{~kg} \mathrm{ha}^{-1}$ aplicado en forma de nitrato de amonio cálcico, $150 \mathrm{~kg}$ $\mathrm{P}_{2} \mathrm{O}_{5}$ ha $^{-1}$ en forma de superfosfato triple, y 30 $\mathrm{kg} \mathrm{K}_{2} \mathrm{O} \mathrm{ha}^{-1}$ como muriato de potasio.

Las parcelas fueron pastoreadas con vacas lecheras secas cada vez que alcanzaban disponibilidad de entrada entre 2200 y $2500 \mathrm{~kg}$ $\mathrm{MS} \mathrm{ha}^{-1}$. La disponibilidad de salida objetivo fue entre 1100 y $1500 \mathrm{~kg} \mathrm{MS} \mathrm{ha}^{-1}$. La disponibilidad de materia seca de pradera, fue determinada indirectamente a través de la medición de la altura comprimida. Se usó un Disco Medidor de Disponibilidad (Rising Plate Meter) de $0,1 \mathrm{~m}^{2}$ de superficie y $300 \mathrm{~g}$ de peso.

Los datos de altura comprimida fueron transformados a disponibilidad de pradera aplicando las formulas de calibración para la zona según cada estación del año (Chile, FIA, 2006).

La fórmula utilizada en primavera fue:

Disponibilidad de forraje $\left(\mathrm{kg}\right.$ MS ha $\left.{ }^{-1}\right)=$ 95*Altura Disco Medidor + 210

La fórmula utilizada en verano fue:

Disponibilidad de forraje ( $\mathrm{kg}$ MS ha-1) = 129*Altura Disco Medidor + 567

\section{Variables Evaluadas.}

Antes de cada pastoreo se procedió a marcar 10 macollos por parcela a los cuales se les midió la altura de lámina extendida y vaina y se hizo la misma medición después de cada pastoreo. También se midió el número de macollos por $\mathrm{m}^{2}$ usando un cuadrante de $0,033 \mathrm{~m}^{2}(11 \times 30 \mathrm{~cm})$.

El pastoreo se realizó con 20 vacas lecheras secas las cuales hacían un uso simultáneo de todas las parcelas. Se midió el número de bocados por minuto en cada tratamiento y la preferencia en pastoreo, contando el número de vacas presentes en cada parcela cada 5 minutos por un periodo de 2 horas según el método utilizado por Balocchi et al. (2000).

Las mediciones se hicieron en 4 fechas: Noviembre y Diciembre, 2005; y Enero y Marzo, 2006.

\section{Variables de la pradera:}

Disponibilidad de pradera en pre $\mathbf{y}$ postpastoreo: Se determinó utilizando un disco medidor de altura comprimida. El disco fue aplicado 50 veces por parcela, antes y después de cada pastoreo.

Densidad de macollos: Después de cada pastoreo, se contó el número de macollos contenidos en un cuadrante de 11 x $30 \mathrm{~cm}(0,033$ $\mathrm{m}^{2}$ ). Esto se realizó 5 veces parcela ${ }^{-1}$.

Largo de lámina: En cada parcela se colocó un transecto de $10 \mathrm{~m}$ de largo, donde se marcaron 10 macollos a $1 \mathrm{~m}$ de distancia, con la ayuda de un clip de color puesto en la base de ellos. En cada macollo se midió el largo de la lámina totalmente expandida, es decir, desde la lígula hasta el extremo distal de la lámina. Esto fue registrado en pre y postpastoreo.

Largo de vaina: En cada macollo marcado se midió, en pre y postpastoreo, el largo de la vaina correspondiente a la lámina descrita anteriormente.

Relación lámina/vaina: Es la relación entre el largo de la lámina y de la vaina en pre y postpastoreo.

\section{Variables del comportamiento animal:}

Consumo aparente: Correspondió a la diferencia entre la disponibilidad de materia seca de la pradera al inicio de un pastoreo y la resultante al término de dicho pastoreo, medidas en kg MS ha-1.

Preferencia en pastoreo: Luego de 10 minutos de iniciado cada pastoreo, cada 5 minutos, durante 2 horas, se registró el número de vacas que pastoreaban cada parcela.

Número de bocados por minuto: En cada parcela se contó durante un minuto el número de bocados que una de las vacas realizaba al pastorear. Esto se realizó 9 veces por parcela y por pastoreo. 
Diseño experimental y análisis estadístico.

Los tratamientos se distribuyeron en tres bloques, de acuerdo a un diseño de bloques completos al azar (Steel et al., 1997).

Se analizó si los datos tenían una distribución normal y homogeneidad en la varianza, requisitos para realizar el ANDEVA. La variable preferencia en pastoreo fue normalizada al transformarse aplicando la función logaritmo natural. El Análisis de Varianza exploró si hubo diferencias entre los tratamientos y se aplicó el test de separación de medias LSD cuando correspondió.

También los datos fueron analizados aplicando el Análisis de Variables Canónicas (CVA).

\section{RESULTADOS}

Variables de la pradera en prepastoreo.

Sólo en Noviembre hubo diferencias significativas en la disponibilidad de materia seca ofrecida dada por los cultivares Quartet AES y AR1 que presentaron los valores extremos de disponibilidad de forraje $(P \leq 0,01)$ (Cuadro 1).

En prepastoreo, el largo de las láminas de los tres tipos de $L$. perenne fue estadísticamente igual $(P>0,05)$ durante todo el período estudiado, al igual que la relación largo de lámina/largo de vaina $(P>0,05)$. Sin embargo, en enero Quartet AES y AR1 presentaron estadísticamente diferentes largos de vaina $(P \leq 0,01)$ (Cuadro 1$)$.

Sólo en Diciembre hubo diferencias estadísticas $(P \leq 0,01)$ en el residuo de pradera dejado por los animales; los mayores residuos fueron para los cultivares Quartet BES y Quartet AES (Cuadro 2).

\section{Variables de la pradera en postpastoreo.}

En el Cuadro 2 se observa que en el largo de lámina postpastoreo hubo diferencias significativas $(P \leq 0,01)$ en los meses de noviembre y enero. En noviembre los cultivares Quartet BES y Quartet AES presentaron los mayores largos de lámina residual, pero en enero fueron los cultivares Quartet AR1 y

Cuadro 1. Disponibilidad de M.S., largo de lámina, largo de vaina y relación lámina vaina en prepastoreo.

Table 1. Herbage mass, lamina length, sheath length and lamina/sheath ratio measured before each grazing event.

\begin{tabular}{cccccc}
\hline Tratamiento & Noviembre & Diciembre & Enero & Marzo & Promedio \\
\hline Disponibilidad pradera prepastoreo & & & & & \\
Quartet BES & & & & \\
Quartet AR1 & $2314 \mathrm{ab}$ & 1963 & 2342 & 2337 & 2239 \\
Quartet AES & $2267 \mathrm{~b}$ & 2235 & 2410 & 2424 & 2334 \\
Significancia & $2433 \mathrm{a}$ & 2106 & 2587 & 2235 & 2340 \\
Quartet BES & $* *$ & n.s. & n.s. & n.s. & n.s. \\
Quartet AR1 & & & & & \\
Quartet AES & 13,4 & 17,4 & 20,7 & 17,4 & 17,23 \\
Significancia & 15,6 & 15,7 & 21,9 & 18,4 & 17,90 \\
Largo lámina prepastoreo (cm) & 14,5 & 18,3 & 25,8 & 17,2 & 18,95 \\
Quartet BES & n.s. & n.s. & n.s. & n.s. & n.s. \\
Quartet AR1 & & & & & \\
Quartet AES & 6,40 & 5,00 & $6,20 \mathrm{ab}$ & 3,33 & 5,23 \\
Significancia & 7,47 & 7,30 & $4,70 \mathrm{~b}$ & 3,37 & 5,71 \\
Largo vaina prepastoreo (cm) & 6,97 & 6,20 & $7,30 \mathrm{a}$ & 3,50 & 5,99 \\
Quartet BES & n.s. & n.s. & $* *$ & n.s. & n.s. \\
Quartet AR1 & & & & & \\
Quartet AES & 2,2 & 4,5 & 3,3 & 5,3 & 3,8 \\
Significancia & 2,1 & 2,5 & 4,8 & 5,7 & 3,8 \\
prepastoreo & 2,3 & 3,0 & 3,4 & 5,0 & 3,4 \\
\hline Relación largo de lámina y vaina & n.s. & n.s. & n.s. & n.s. & n.s. \\
\hline
\end{tabular}

${ }^{1}$ Tratamientos: Quartet BES Quartet Bajo Endófito Estandar; Quartet AR1Quartet Bajo Endófito AR1; Quartet AES Quatet Alto Endófito Estandar.

$2 * * P \leq 0,01$; n.s.: No significativo $(P>0,05)$ 
Cuadro 2. Disponibilidad de M.S., largo de lámina, largo de vaina, relación lámina vaina y número de macollos en postpastoreo.

Table 2. Herbage mass, lamina length, sheath length, lamina/sheath ratio and tiller number measured after each grazing event.

\begin{tabular}{|c|c|c|c|c|c|}
\hline Tratamiento & Noviembre & Diciembre & Enero & Marzo & Promedio \\
\hline \multirow{2}{*}{\multicolumn{6}{|c|}{ Pradera residual postpastoreo_(kg }} \\
\hline & & & & & \\
\hline Quartet BES $^{1}$ & 1211 & $1510 \mathrm{a}$ & 1375 & 1247 & 1336 \\
\hline Quartet AR1 & 1143 & $1344 b$ & 1542 & 1082 & 1278 \\
\hline Quartet AES & 1174 & $1390 \mathrm{ab}$ & 1697 & 1133 & 1349 \\
\hline Significancia $^{2}$ & n.s. & $* *$ & n.s. & n.s. & n.s. \\
\hline \multicolumn{6}{|c|}{ Largo lámina postpastoreo $(\mathrm{cm})$} \\
\hline Quartet BES & $2,77 \mathrm{a}$ & 4,43 & $3,00 \mathrm{~b}$ & 4,20 & 3,60 \\
\hline Quartet AR1 & $0,33 b$ & 4,97 & $4,30 \mathrm{ab}$ & 2,40 & 3,00 \\
\hline Quartet AES & $1,83 \mathrm{ab}$ & 2,33 & $7,10 \mathrm{a}$ & 2,80 & 3,52 \\
\hline Significancia & $* *$ & n.s. & $* *$ & n.s. & n.s. \\
\hline \multicolumn{6}{|c|}{ Largo vaina postpastoreo $(\mathrm{cm})$} \\
\hline Quartet BES & 5,07 & 5,57 & 6,80 & 2,73 & 5,04 \\
\hline Quartet AR1 & 4,57 & 4,23 & 4,30 & 2,97 & 4,02 \\
\hline Quartet AES & 4,63 & 3,73 & 7,10 & 2,73 & 4,55 \\
\hline Significancia & n.s. & n.s. & n.s. & n.s. & n.s. \\
\hline \multicolumn{6}{|c|}{$\begin{array}{l}\text { Relación largo de lámina y vaina } \\
\text { postpastoreo }\end{array}$} \\
\hline Quartet BES & $0,88 \mathrm{a}$ & 0,65 & $0,54 \mathrm{ab}$ & 1,56 & 0,91 \\
\hline Quartet AR1 & $0,09 b$ & 1,12 & $0,32 b$ & 0,82 & 0,59 \\
\hline Quartet AES & $0,40 \mathrm{ab}$ & 0,61 & $0,99 a$ & 0,96 & 0,74 \\
\hline Significancia & $* *$ & n.s. & $* *$ & n.s. & n.s. \\
\hline \multicolumn{6}{|l|}{ Número de macollos $\mathrm{m}^{-2}$} \\
\hline Quartet BES & 3410 & 3505 & 2969 & $2131 b$ & 3004 \\
\hline Quartet AR1 & 3063 & 3020 & 2134 & $2959 a$ & 2794 \\
\hline Quartet AES & 2973 & 2384 & 2202 & $2535 \mathrm{ab}$ & 2524 \\
\hline Significancia & n.s. & n.s. & n.s. & $* *$ & n.s. \\
\hline
\end{tabular}

${ }^{1}$ Tratamientos: Quartet BES Quartet Bajo Endófito Estandar; Quartet AR1 Quartet Bajo Endófito AR1; Quartet AES Quatet Alto Endófito Estandar.

$2 * * P \leq 0,01 ;$ n.s. No significativo $(P>0,05)$.

Quartet AES.

No hubo diferencias significativas $(P>0,05)$ en el largo de la vaina residual en ninguno de los tratamientos. Sin embargo, en los meses de Noviembre y Enero, la relación largo de lámina/ largo de vaina residual fue estadísticamente diferente $(P \leq 0,01)$. En Noviembre la relación largo de lámina/largo de vaina residual fue mayor para el cultivar Quartet BES que para Quartet AR1, mientras que en Enero ésta fue mayor para el cultivar Quartet AES en relación a Quartet AR1 (Cuadro 2).

La densidad de macollos sólo fue estadísticamente diferente $(P<0,01)$ durante Marzo (Cuadro 2). El cultivar Quartet AR1 presentó la mayor densidad de macollos, mientras que Quartet BES la menor.

Variables del comportamiento animal.

En relación al comportamiento animal, las evaluaciones de Noviembre, Diciembre y para el ANDEVA al promedio de los períodos, mostró que hubo diferencias estadísticas $(P \leq 0,01)$ entre los tipos de L. perenne. En forma consistente el cultivar Quartet BES fue el menos consumido. En Enero y Marzo no hubo diferencias significativas $(P>0,05)$ entre los tipos de L. perenne (Cuadro 3).

La tasa de bocados no mostró diferencias estadísticas $(P>0,05)$ entre los tipos de $L$. perenne, ni tampoco el análisis de la preferencia 
Cuadro 3. Efecto del nivel y tipo de hongo endófito sobre el consumo aparente, tasa de bocados y preferencia en pastoreo en vacas lecheras.

Table 3. Effect of the type and level of endophyte fungi on the apparent intake, bite rate and grazing preference of dairy cows.

\begin{tabular}{cccccc}
\hline Tratamiento & Noviembre & Diciembre & Enero & Marzo & Promedio \\
\hline $\begin{array}{c}\text { Consumo aparente } \\
\text { (kg materia seca por ha }^{-1} \text { ) }\end{array}$ & & & & & \\
Quartet BES $^{1}$ & $1103 \mathrm{~b}$ & $453 \mathrm{~b}$ & 967 & 1090 & $903 \mathrm{~b}$ \\
Quartet AR1 $_{\text {Quartet AES }}$ & $1124 \mathrm{~b}$ & $892 \mathrm{a}$ & 868 & 1342 & $1057 \mathrm{a}$ \\
Significancia $^{2}$ & $1258 \mathrm{a}$ & $716 \mathrm{ab}$ & 890 & 1102 & $992 \mathrm{ab}$ \\
(** & ** & n.s. & n.s. & $* *$ \\
\hline Tasa de bocados & & & & & \\
(número bocados minuto $^{-1}$ ) & 53 & 53 & 54 & 58 & 55 \\
Quartet BES & 52 & 58 & 54 & 62 & 57 \\
Quartet AR1 & 54 & 55 & 54 & 63 & 57 \\
Quartet AES & n.s. & n.s. & n.s. & n.s. & n.s. \\
Significancia & & & & & \\
Quartet BES & 3,03 & 2,67 & 2,13 & $1,53 \mathrm{~b}$ & 2,34 \\
Quartet AR1 & 2,03 & 2,53 & 2,63 & $1,77 \mathrm{ab}$ & 2,24 \\
Quartet AES & 2,23 & 2,23 & 1,93 & $2,00 \mathrm{a}$ & 2,10 \\
Significancia & & & & & \\
\hline Preferencia en pastoreo & & & & & \\
\hline
\end{tabular}

${ }^{1}$ Tratamientos: Quartet BES Quartet Bajo Endófito Estandar; Quartet AR1 Quartet Bajo Endófito AR1; Quartet AES Quatet Alto Endófito Estandar.

$2 * * P \leq 0,01 ;$ n.s. No significativo $(P>0,05)$

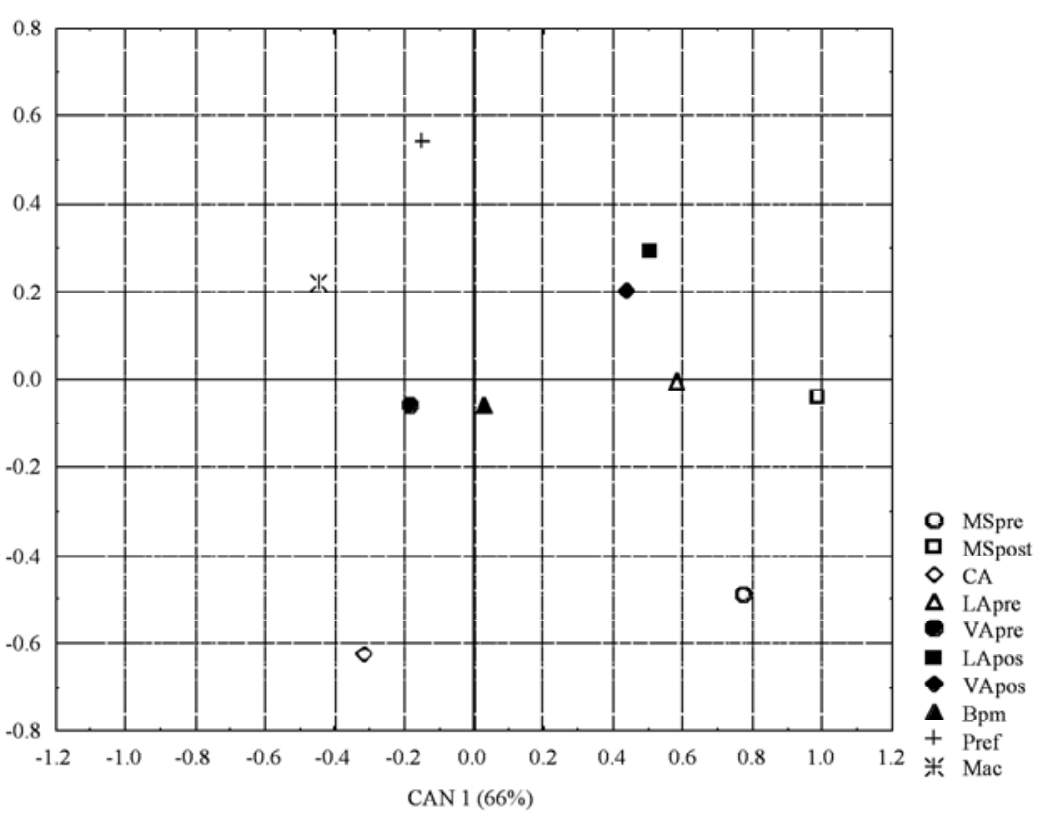

Figura 1. Relación entre las variables evaluadas de la pradera y del pastoreo.

Figure 1. Relationships between the pasture and grazing animal variables.

MSpre: materia seca prepastoreo, MSpost: materia seca postpastoreo, CA: consumo aparente, LApre: lámina prepastoreo, VApre: vaina prepastoreo, LApost: lámina postpastoreo, VApost: vaina postpastoreo, Bpm: bocados por minuto, Pref: preferencia en pastoreo, Mac: número de macollos $\mathrm{m}^{-2}$. 


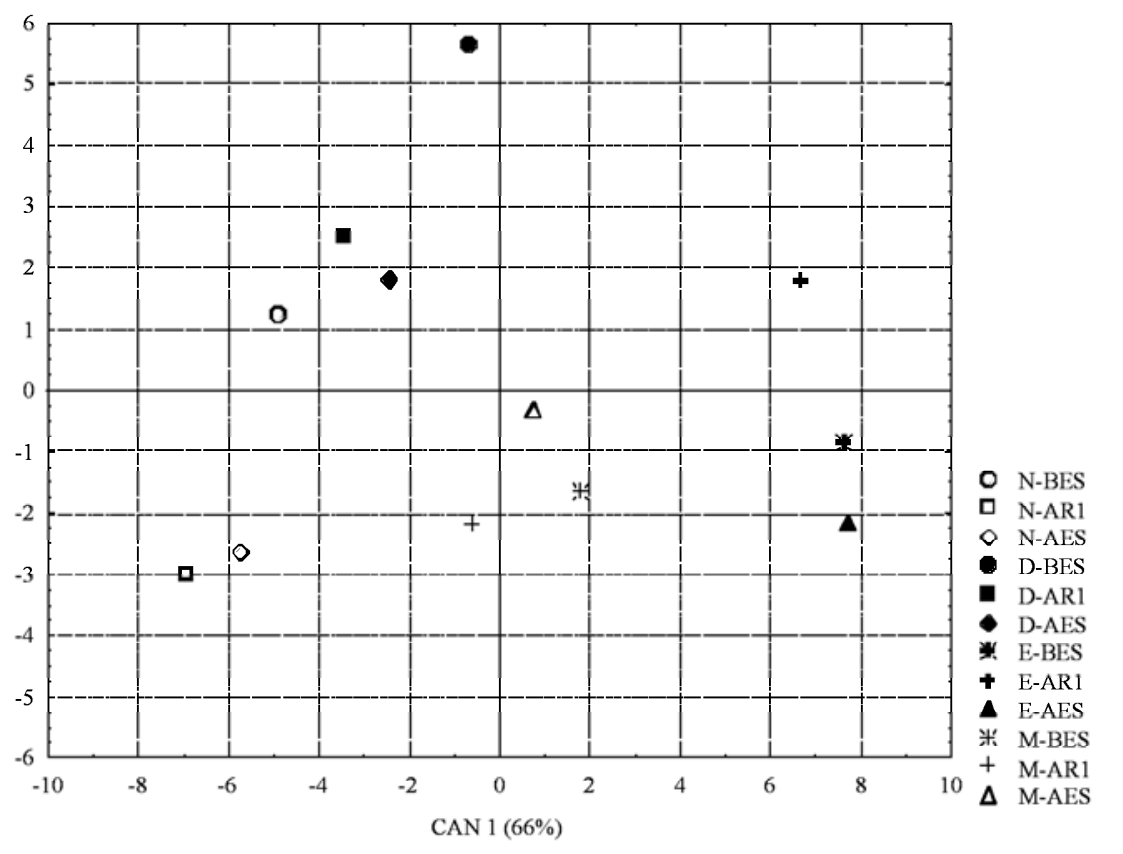

Figura 2. Distribución espacial de los tratamientos según cada fecha de pastoreo.

Figure 2. Spatial distribution of the treatments according to the grazing dates.

N-BES: noviembre-bajo endófito estandar, N-AR1: noviembre AR1, N-AES: noviembre, alto endófito estandar, D-BES: diciembre, bajo endófito estandar, D-AR1: diciembre AR1, D-AES: diciembre, alto endófito estandar, E-BES: enero, bajo endófito estandar, E-AR1: enero AR1, E-AES: enero, alto endófito estandar, M-BES: marzo, bajo endófito estandar, M-AR1: marzo,AR1, M-AES: marzo, alto endófito estandar.

en pastoreo $(P>0,05)$, salvo en el mes de Marzo $(P \leq 0,01)$ donde los cultivares Quartet AR1 y Quartet AES fueron preferidos (Cuadro 3).

\section{Análisis de Variables Canónicas.}

El Análisis de Variables Canónicas (AVC) exploró las relaciones entre las variables evaluadas, los tratamientos y fechas, e indicó diferencias significativas (Wilk's Lambda $P<0,001)$ entre ellas. Las dos primeras variables canónicas explicaron el $82 \%$ de las diferencias medidas (Figura 1): 66\% explicado por la primera variable canónica (CAN 1) y $17 \%$ explicado por la segunda variable canónica (CAN 2).

En CAN 1, la disponibilidad de pradera, tanto en prepastoreo como en postpastoreo, fueron las variables más importantes en explicar las diferencias entre los tipos de L. perenne, comportamiento animal y fechas de pastoreo. Estas variables fueron positivamente influidas por el largo de lámina y vaina en prepastoreo, y el largo de lámina en postpastoreo. Hubo un contraste (relación inversa) entre la disponibilidad de pradera en prepastoreo y la cantidad de macollos por $\mathrm{m}^{2}$ (Figura 1).

En CAN 2 las diferencias más relevantes entre los tipos de $L$. perenne fueron explicadas por el contraste entre la preferencia en pastoreo con el consumo aparente y la materia seca prepastoreo. Cuando las vacas lecheras aumentaron el consumo de pradera en pastoreo, la preferencia ejercida por uno $\mathrm{u}$ otro cultivar disminuyó (Figura 1).

El CVA para CAN 1 separó a los tipos de L. perenne de acuerdo a las diferentes fechas evaluadas (Figura 2). La disponibilidad de pradera en prepastoreo y la masa residual de pradera aumentó de la siguiente manera: Noviembre < Diciembre < Marzo < Enero. De igual manera variaron el largo de lámina y de vaina en prepastoreo, y el largo de lámina en postpastoreo. De esta forma, en enero se midió una mayor cantidad de láminas y vainas sin consumir, mientras que en noviembre hubo un 
mayor consumo de pradera unido a una mayor densidad de macollos, los que contaban con un menor desarrollo foliar.

CAN 2 discriminó entre los tipos de $L$. perenne dentro de cada fecha. En los pastoreos de noviembre y diciembre BES fue el más preferido; en el pastoreo de enero lo fue AR1; mientras que en marzo no hubo una discriminación entre BES, AES y AR1 (Figura 2).

\section{DISCUSIÓN}

\section{Atributos de la pradera en prepastoreo.}

En general los atributos de las praderas (Cuadro 1) como el largo de lámina, el largo de vaina y la relación lámina/vaina, además de la disponibilidad de materia seca en prepastoreo fueron estadísticamente iguales entre los tipos de $L$. perenne. Cuando hubo diferencias estadísticas, éstas fueron en casos puntuales: En noviembre para la disponibilidad de pradera prepastoreo y en enero para el largo de vaina. Características como largo de lámina y relación lámina/vaina fueron estadísticamente similares durante toda la evaluación (Cuadro 1). Se considera que la homogeneidad medida era esperable al tratarse de un mismo cultivar (Quartet), y se desprende que los tipos de hongo endófito no alteraron, al menos, la expresión génica de las características de crecimiento del cultivar Quartet evaluadas. Estos resultados son coherentes con lo reportado por Bluett et al. (2005), que indican que la presencia de distintos tipos de endófito (endófito standard, AR1 y sin endófito) no generaron diferencias en tasa de crecimiento, producción anual de materia seca, densidad de macollos y composición botánica en $L$. perenne.

Otras investigaciones han reportado que el hongo endófito estimularía en $L$. perenne un aumento en el rendimiento de las praderas (Prestidge et al., 1982; Latch et al., 1985; Torres, 1996; Popay et al., 1999; Easton et al., 2001; Rattray, 2003), un mayor vigor de plantas, mayor índice de área foliar, mayor número de macollos y pseudotallos, mayor desarrollo radicular (Hesse et al., 2003; Hahn, 2008), y mayor tolerancia a sequía (Prestidge et al., 1982; Easton et al., 2001; Hahn, 2008). Si bien estos trabajos muestran cambios que L. perenne puede experimentar debido a la presencia del hongo endófito, el presente estudio evidencia que el cultivar Quartet no expresaría mayores cambios al ser contrastados diferentes tipos de hongo endófito.

\section{Atributos de la pradera en postpastoreo.}

Las diferencias en el largo de lámina residual mostraron un contraste entre los tipos de hongo endófito (Cuadro 3). El análisis univariado indicó que la longitud de la lámina en prepastoreo fue estadísticamente igual entre los tipos de $L$. perenne (Cuadro 2), pero no así en postpastoreo (Cuadro 3). Esto sugiere que probablemente las vacas ejercieron una discriminación ante los materiales ofrecidos, a nivel de lámina. Así en noviembre el largo de lámina en postpastoreo fue menor en Quartet AR1 y AES, mientras que en enero fue para Quartet BES y AR1. De esta forma, existiría una interacción entre el tipo de hongo endófito y las condiciones ambientales de cada época, ocurriendo probablemente cambios a nivel bioquímico en las plantas que determinarían la selección por las vacas lecheras.

Los resultados sugieren que las vacas lecheras pueden mostrar preferencia al pastorear un mismo cultivar con diferente tipo de hongo endófito, discriminando según la naturaleza del hongo endófito. La selección se puede ejercer en forma aún más fina, verificándose por la remoción de algunas partes de las plantas por sobre otras (Hodgson, 1979), como por ejemplo, la selección a favor de las láminas por sobre los tallos (Holmes, 1980; Stuth, 1991; Schewmaker et al., 1997; Hodgson y Brookes, 1999). De esto la importancia de realizar mediciones finas, como las realizadas en este estudio que permitieron medir el proceso de preferencia ocurrido, por el que las vacas lecheras discriminaron un mismo cultivar según la época del año y el tipo y nivel de hongo endófito.

\section{Variables del comportamiento animal.}

El consumo de pradera en pastoreo, se relaciona al tiempo de pastoreo, la tasa de bocado (número de bocados por minuto) y al tamaño de cada bocado (Hodgson, 1990). Así, las vacas lecheras pastorean generalmente de 
6 a 9 horas por día, raramente pastorean más de 9 horas al día; y el número de bocados por minuto no varía considerablemente: de 55 a 65 bocados. El consumo de pradera por bocado es muy sensible a cambios en la altura de la pradera (Phillips y Denne, 1988; Hodgson, 1990) y densidad del forraje (Phillips y Denne, 1988); pudiendo ser compensados, hasta cierto límite, por un aumento en la tasa de bocado y/o en el tiempo de pastoreo (Hodgson y Brookes, 2004).

En el presente estudio, el consumo aparente de pradera fue similar entre los tres tratamientos durante enero y marzo (Cuadro 3). Se sugiere que la baja condición de estrés hídrico durante el verano en que se desarrolló el estudio, evitó una diferenciación en la expresión bioquímica de los hongos endófitos, lo que probablemente ayudó a que no hubiera una mayor discriminación de los tipos de L. perenne ofrecidos en pastoreo. La sequía incrementa el nivel de toxinas generadas por el hongo endófito, en especial en endófitos tipo AES, las que son contrarias al pastoreo. Al contrario, condiciones ambientales normales para el crecimiento de $L$. perenne atenúan la generación de las toxinas por el hongo endófito (Ravel et al., 1995; Oliveira et al., 2004).

El Análisis de Variables Canónicas (AVC) analizó en forma integrada todas las variables evaluadas y fue capaz de detectar diferencias estadísticas significativas, entre fechas y entre los tipos de L. perenne, en relación al comportamiento de los animales y a las variables evaluadas de la pradera (Figuras 1 y 2). De esta forma, el AVC indicó que la disponibilidad de pradera y el largo de lámina, ambos en pre y postpastoreo, fueron las variables que más explicaron las diferencias entre tratamientos (Figura 1). Estas se relacionaron con el consumo de pradera de la siguiente manera: Las variables de prepastoreo con las características de lo ofrecido, y las variables de postpastoreo con el resultado del consumo. La oferta, en términos de láminas, vainas y pradera ofrecida, dentro de cada fecha de evaluación, fue bastante homogénea; pero las diferencias medidas postpastoreo entre los tratamientos, correspondieron a un consumo diferenciado de las praderas (Figuras 1 y 2).

El AVC mostró un cambio en el comportamiento de las vacas al pastorear $L$. perenne cv. Quartet de acuerdo al tipo de endófito y la época del pastoreo, la que se expresó de la siguiente manera: En noviembre y en diciembre el consumo aparente fue $\mathrm{QBES}>\mathrm{QAR} 1=\mathrm{QAES}$, en enero QAR1>QBES=QAES, y en marzo no hubo diferencias (Figuras 1 y 2). El mayor consumo de QBES, en ambos meses, se asoció a un incremento de los bocados por minuto, lo que ocurrió en fechas sucesivas. Estos resultados en apariencia se contraponen a los entregados por ANDEVA, la que indicó que en noviembre y diciembre QAES fue el más consumido. Sin embargo, se interpreta que ambos análisis indican que la preferencia y cantidad consumida se pueden ser variables contrapuestas, estando la cantidad consumida estrechamente relacionada al manejo del pastoreo (Tharmaraj et al., 2003), mientras que la preferencia al animal (Hodgson, 1979). Por otra parte, la aparente discordancia de ANDEVA y CVA ha sido analizada por Matthew et al. (1994) en relación a los análisis multivariados. Cuando en el análisis de datos el objetivo es explorar interacciones, relaciones o comportamientos, el AVC se hace más apropiado que el ANDEVA, ya que ésta última analiza en forma independiente cada variable, mientras que el AVC las analiza todas en forma integrada.

El endófito AR1 en L. perenne presenta la característica que no produce alcaloides que pueden generar problemas para el ganado, como son el lolitrem B y la ergovalina, que sí son producidos por el endófito estándar (Rattray, 2003). Durante el estado vegetativo el hongo endófito ha permanecido muy cercano a la base de las plantas de gramínea. La fase reproductiva en la gramínea se caracteriza por la elongación del tallo que generará la inflorescencia; cuando esto ocurre, el hongo endófito se prolonga junto con el tallo, y así la distribución de los alcaloides dentro de la planta varía, los que se concentrarán en la inflorescencia (Ball et al., 1995; Keogh et al., 1996; Lane et al., 1997). Probablemente, la preferencia en pastoreo mostrada por las vacas lecheras por QAR1 en enero fue el reflejo de la distribución hacia la superficie de la pradera del hongo endófito y sus alcaloides, lo que generó una expresión positiva, en términos de preferencia por el ganado, hacia endófito AR1 
(Figura 1).

Los resultados entregados por AVC, que al evaluar integradamente todas las variables medidas y permitió detectar con mayor claridad los cambios en el comportamiento ingestivo de las vacas lecheras, concuerdan con trabajos que sostienen que $L$. perenne con endófitos nobles son más consumidos que aquellos con endófito natural equivalente a AES (Butendieck et al., 1994; Balocchi y Teuber, 2003; Fraser y Goulter, 2006). Se sugiere que, cuando se trata de un mismo cultivar, las características morfológicas de las plantas individuales no serían determinantes en la preferencia de las vacas en pastoreo por uno u otro tipo de $L$. perenne, como se aprecia en el Cuadro 1 , sino que serían diferencias que se explicarían por atributos endógenos a cada tipo de L. perenne usado. Estos tendrían su génesis en el tipo de hongo endófito contenido y a la fecha de evaluación, expresada por el estado fenológico (Figuras 1 y 2). La liberación de productos que el huésped o el hospedero pudieran liberar al medio celular de la planta, generando sabores y/u olores, que tendrían un efecto directo en el comportamiento ingestivo del animal.

\section{CONCLUSIONES}

Los atributos morfológicos de L. perenne no fueron afectados por el tipo o nivel de hongo endófito.

El comportamiento de las vacas lecheras en pastoreo fue alterado por el tipo de hongo endófito contenido y la época del año. Las vacas lecheras fueron capaces de ejercer preferencia al discriminar entre los tipos del cultivar Quartet, explicada por la interacción entre el ambiente (época del año que definió el estado fenológico de las plantas) y atributos endógenos de las plantas, como fueron en este caso el tipo hongo endófito usado.

La expresión génica de AR1 mostró una ventaja sobre AES y BES sólo durante el verano, a través de una mayor preferencia en pastoreo por las vacas lecheras.

\section{BIBLIOGRAFIA}

BALOCCHI, O.; TEUBER, N. 2003. Recursos forrajeros en producción de leche. II Novedades en gramíneas y leguminosas forrajeras. In: Teuber, N.; Uribe, H.; Opazo, R. (eds.). Hagamos de la lechería un buen negocio. Instituto de Investigaciones Agropecuarias. Serie Actas Inia No 24. pp 13-19.

BALOCCHI, O.; LÓPEZ, I.; ISLA, F. 2000. Evaluación de cultivares de Lolium perenne bajo pastoreo con vacas lecheras. Monografía. Universidad Austral de Chile - Agritec - Anasac - SG 2000. Valdivia. Chile. 23 p.

BALL, O. J. P.; PRESTIDGE, R.A.; SPROSEN, J. M. 1993. Effect of plant age and endophyte viability on peramine and lolitrem $\mathrm{B}$ in perennial ryegrass seedlings. Proceedings of the 2nd International Symposium on Acremonium/Grass interactions. Eds. Hume, D.E., Latch, G.C.M., Easton H.S. AgResearch, Palmerston North, New Zealand. pp 63-65.

BALL, O. J. P.; PRESTIDGE, R. A.; SPROSEN, J. M. 1995. Interrelationships between Acremonium lolii peramine and lolitrem B in perennial ryegrass. Applied and environmental Microbiology 61: 1527-1533.

BLUETT S. J.; THOM, E. R.; CLARK, D. A, MACDONALD, K. A.; MINNEE, E. M. K. 2005. Effects of perennial ryegrass infected with either AR1 or wild endophyte on dairy production in the Waikato. New Zealand Journal of Agricultural Research 48: 197-212.

BUTENDIECK, N.; ROMERO, O.; HAZARD, S.; MARDONES, P.; GALDAMES, R. 1994. Caída del consumo y producción de leche en vacas alimentadas con Lolium perenne, infectada con Acremonium lolii. Agricultura Técnica (Chile) 54:1:6.

CHILE, FONDO PARA LA INNOVACION AGRARIA (FIA). 2006. Validación y difusión de mejores prácticas de pastoreo para el sur de Chile. Osorno. Chile. Inf. Técnico $N^{\circ} 3$. 2p.

CHILE, INIA (Instituto de Investigaciones Agropecuarias).1985. Suelos Volcánicos de Chile. Juan Tosso (ed.) Primera edición.Talleres Gráficos del INIA. Santiago. Chile. 723p.

EASTON, H. S.; CHRISTENSEN, M. J.; EERENS, J. P. J.; FLETCHER, L. R.; HUME, D. E.; KEOGH, R. G.; LANE, G. A.; LATCH, G. C. M.; PENNELL, C. G. L.; POPAY, A. J.; ROLSTON, M. P.; SUTHERLAND, B. L.; TAPPER, B. A. 2001. Ryegrass endophyte: a New Zealand Grassland success story. Proceedings of the New Zealand Association 63: 37-46.

FLETCHER L. R. 1999. "Non-toxic" endophytes 
in ryegrass and their effect on livestock health and production. In: Woodfield D. R.; Matthew C. (eds.). Ryegrass endophyte: an essential New Zealand Symbiosis. Proceedings of the New Zealand Grassland Asociation. Palmerston North, New Zealand. pp. 133-140.

FLETCHER, L. R.; HARVEY, I. C. 1981. An association of a Lolium endophyte with ryegrass staggers. New Zealand Veterinary Journal 29: 185186.

FRASER, T.; GOULTER, C. 2006. Deciphering the letterbox forage species leaflets. Proceedings of the SIDE 17: 170-177.

GLENN, A. E.; BACON, C. W. 1997. Distribution of ergot alkaloids within the amily Clavicipitaceae. In: Neotyphodium/Grass Interactions. Bacon, C.W. and Hill, N.S., eds., Plenum Press, New York. pp. 53-56.

HAHN, H.; MCMANUS, M. T.; WARNSTORFF, K.; MONAHAN, B. J.; YOUNG, C. A.; DAVIES, E.; TAPPER, B. A.; SCOTT, B. 2008. Neotyphodium fungal endophytes confer physiological protection to perennial ryegrass (Lolium perenne $\mathrm{L}$.) subjected to a water deficit. Environmental and Experimental Botany 63: 183-199.

HESSE,U.;SCHÖBERLEIN, W.; WITTENMAYER, L.; FÖRSTER, K.; WARNSTORFF, K.; DIEPENBROCK, W.; MERBACH, W. 2003. Effects of Neotyphodium endophytes on growth, reproduction and drought-stress tolerance of three Lolium perenne L. genotypes. Grass and Forage Science 58: 407 - 415.

HODGSON, J. 1979. Nomenclature and definitions in grazing studies. Grass and Forage Science 34: $11-18$

HODGSON, J. 1990. Grazing management. Science into practice. Oxford, Blackwell Science 203 p.

HODGSON, J.; BROOKES, J. 1999. Nutrition of grazing animals. In. J.White y Hodgson (eds.). New Zealand Pasture and crop science. Oxford University. pp. 117-132.

HOLMES, W. 1980. Grazing Management . In. W. Holmes (ed.) Grass, its production and utilization. The British Grassland Society. Blackwell Scientific Publications. Londres. Inglaterra. pp 125-173.

KEOGH, R. G.; TAPPER, B. A.; FLECHTER, R. H. 1996. Distribution of fungal Endophyte Acremonium lolii, and of the alkaloids lolitrem $\mathrm{B}$ and peramine, within perennial ryegrass. New Zealand Journal of Agricultural Research 39: 121127.

LANE, G. A.; TAPPER, B. A.; DAVIES, E.; HUME, D. E.; LATCH, G. C. M.; BARKER, D. J.; EASTON, H. S.; ROLSTON, M. P. 1997. Effect of grow conditions on alkaloids concentrations in perennial ryegrass naturally infected with endophyte. In: Bacon, C.W., Hill, N.S. (eds.) Neotyphodium/grass Interactions. Plenium Press. New York y London. pp 179-182.

LATCH, G. C. M.; HUNT, W. F.; MUSGRAVE, D. R. 1985. Endophytic fungi affect growth of perennial ryegrass. New Zealand Journal of Agriculture Research 28: 165-168.

MATTHEW, C.; LAWOKO, C. R. O.; KORTE, C. J.; SMITH, D. 1994. Application of canonical discriminant analysis, principal component analysis, and canonical correlation analysis as tools for evaluating differences in pasture botanical composition. New Zealand Journal of Agricultural Research 37: 509-520

MORTIMER, P. H.; Di MENNA, M. 1983. Ryegrass staggers: further substantiation of Lolium endophyte aetiology and the discovery of weevil resistance of ryegrass pastures infected with Lolium endophyte. Proceedings of the New Zealand Grassland Association 44:240-243.

OLIVEIRA, J. A.; GONZALEZ, E.; CASTRO, P.; COSTAL, L. 2004. Efectos de la infección con un hongo endófito sobre la producción de materia seca, persistencia y valor nutritivo en raygrass inglés en Galicia (Noroeste de España). Spanish Journal of Agricultural Research 4: 558-563.

PHILLIPS, C. J. K.; DENNE, S. K. 1998. Variation in the grazing behaviour of dairy cows measured by vibrorecorder and bite count monitor. Applied Animal Behaviour Science 21: 325-329.

POPAY, A. J.; ROWAN, D. D. 1994. Endophyte fungi as mediators of plant-insect interactions. In: Bernays, E.A. (ed.) Insect-plant Interactions. E.A CRC Press. pp 83-103

POPAY, A. J.; HUME, D.E.; BALTUS, J. G.; LATCH, G. C. M.; TAPPER, B. A.; LYONS, T. B. COOPER, B. M.; PENNELL, C. G.; EERENS, J. P. J.; MARSHALL, S. L. 1999. Field performance of perennial ryegrass (Lolium perenne) infected with toxin-free fungal endophytes (Neotyphodium spp). Ryegrass endophyte: an essential New Zealand Symbiosis. Grassland Research and Practice Series. 7:113-122.

PRESTIDGE, R. A.; BALL, O. J. P. 1993. The rol of endophytes in alliviating plant biotic stress. In: New Zealand Proceeding of the 2nd International Symposium on Acremonium/grass Interactions: plenary papers. Hume, D.E.; Latch, G.C.M., Easton, H.S. (eds.). AgResearch Palmerston North. New Zealand. pp 141-151.

PRESTIDGE, R. A.; POTTINGER, R. P.; BARKER, G. M. 1982. An association of Lolium endophyte with raygrass resistance to Argentine stem weevil. Proceeding of the 35th New Zealand Weed and 
Pests Control Conference. pp 119-122.

RATTRAY, P. V. 2003. Ryegrass endophyte: An Upto-Day review of its effects. Report produced by meal and wool innovation Ltd. under contract to Merino New Zealand Inc. New Zealand. 36 p.

RAVEL, C. ; CHARMET, G. ; BALFOURIER, F. 1995. Influence of the fungal endophyte Acremonium lolii on agronomic traits of perennial ryegrass in France. Grassand forage Science 50: 75-80.

SCHEWMAKER, G.; MAYLAND, H.; HANSEN, S. 1997. Cattle grazing preference among eight endophyte-free tall fescue cultivars. Agronomy Journal 89: 695-701.

STEEL R. G. D.; TORRIE J. H.; DICKEY D. A. 1997 Principles and procedures of statistics: A biometrical approach. New York: McGraw-Hill. $688 \mathrm{p}$.

STEWART, A.V. 1985. Perennial ryegrass seedling resistance to Argentine stem weevil. New Zealand
Journal of Agricultural Research 28: 403-407.

STUTH, J. 1991. Forage behaviour. In. Heitschmidt, ? Stuth,J. (eds.). Grazing management: an ecological perspective. Portland, Oregon, USA. Timber Press. pp 65-84.

TORRES, A. 1996. Los endófitos (Acremonium sp) y su incidencia en la ganadería de la zona sur. In: Latrille, L. (ed.) Producción Animal. Valdivia. Universidad Austral de Chile. Facultad de Ciencias Agrarias. Instituto de Producción Animal. pp: 5163.

THARMARAJ, J.; WALES, W. J.; CHAPMAN, D. F.; EGAN, A. R. 2003. Defoliation pattern, foraging behaviour and diet selection by lactating dairy cows in response to sward height and herbage allowance of a ryegrass-dominated pasture. Grass and Forage Science. 58: 225-238.

WRIGHTSON SEEDS. 2007. Understanding endophytes. Forage Focus. $\mathrm{N}^{\circ}$ 5. New Zealand. $2 p$. 